\title{
Forensic Pathology
}

\section{'HUDS': HUNTER UNEXPECTED DEATH SURVEILLANCE}

Leah J. Clifton, Allan D. Cala

Department of Forensic Medicine Newcastle, Forensic and Analytical Science Service, NSW Pathology, NSW, Australia

Despite advances in diagnosis and treatment, infectious disease causes significant mortality worldwide. Infection also remains one of the most common unexpected findings at autopsy and the autopsy remains an important tool in the diagnosis of sudden infectious deaths and the demonstration of disease pathogenesis. In April 2014 a 'HUDS' working group was established in Newcastle, New South Wales, in order to survey sudden unexpected deaths of suspected infectious causes in the Hunter New England region of New South Wales. This involved collaborative input from the Department of Forensic Medicine in Newcastle, the Hunter New England Population Health Unit and the Hunter Area Pathology Microbiology Unit. The objectives of the group were to develop an approach to the accurate and early detection of infectious deaths at post-mortem examination which may be of public health interest with the view to identifying possible clusters of infection outbreaks. The proposal was based on the 'UNEX' and the 'Med-X' population based surveillance systems utilised in the United States of America. An overview of the 'HUDS' initiative, the documents developed, the challenges arising from our experience and the data collected over a 21 month period will be presented along with key case examples.

\section{SUDI: A MODERN APPROACH}

Bianca Phillips

Forensic and Scientific Services, Health Support Queensland, Queensland Department of Health, Qld, Australia

Infants are a vulnerable group due to their reliance on caregivers to provide them with the necessities of life. This vulnerability is further complicated by their inability to communicate, their immature homeostatic mechanisms and their propensity to deteriorate suddenly in the face of illness. These factors make it extremely important for unexpected infant deaths to receive a high level of investigation in order to recognise unnatural deaths, and to attribute a cause of death in as many cases as possible. Sudden unexpected death in infancy (SUDI) is a term that refers to all sudden and unexpected deaths in infants. The minimum level of investigation required in these deaths is made up of three components: scene investigation, review of the clinical history and post-mortem examination. Adequate investigation is also required for the death to be attributed to sudden infant death syndrome (SIDS) in some cases where a recognisable cause of death cannot be identified.
There is currently no universal standard protocol for the investigation of SUDI. This talk will briefly summarise current approaches to investigation and outline a range of emerging techniques that are making their way into daily practice.

\section{FENTANYL-RELATED DEATHS: A 5-YEAR RETROSPECTIVE STUDY (2010-2014, 81 CASES) IN THE DEPARTMENT OF FORENSIC MEDICINE, NEWCASTLE, FORENSIC AND ANALYTICAL SCIENCE SERVICE}

$\underline{\text { Rexson Tse }}$

Department of Forensic Medicine Newcastle, Forensic and Analytical Science Service, NSW, Australia

Background: Fentanyl is a synthetic opioid developed in the 1960 s and is 50-100 times more potent than morphine. Fentanyl is recognised to be abused in the community. The Department of Forensic Medicine Newcastle, Forensic and Analytical Science Service (DOFM, Newcastle) has recently experienced an increase in fentanyl related deaths. This study was carried out to characterise fentanyl related death in DOFM, Newcastle.

Method: A retrospective study (5 years, 2010-2014) of deaths related to fentanyl in DOFM, Newcastle, was carried out noting the age, gender, cause of death, toxicological analysis, mode of delivery, and presumed mechanism of death.

Result: There was a striking increase in death related to fentanyl from 1 case in 2010 to 38 cases in $2014(n=81)$. Most of the fatal cases had a fentanyl blood level between $0.01-0.029 \mathrm{mg} / \mathrm{L}$ with the highest level being $0.08 \mathrm{mg} / \mathrm{L}$. The most common cause of death was mixed drug toxicity. The victims were mostly middle aged males and the most common mechanism of death was respiratory depression. Other substances detected apart from fentanyl were benzodiazepine, opioids and alcohol. The most common route of administration was via extracting fentanyl from a patch and injecting it intravenously.

\section{FORENSIC AND AUTOPSY NEUROPATHOLOGY WORKSHOP}

Clive Cooke $^{1}$, Alanah Buck ${ }^{1}$, Vicki Fabian

${ }^{1}$ Forensic Pathology, and ${ }^{2}$ Neuropathology, PathWest, Perth, WA, Australia

Twenty (20) cases and questions, some in several parts, will excite the participants in the form of a Workshop Quiz. The cases and questions have been carefully selected as being noteworthy for their importance, and will challenge everyone's diagnostic abilities. For trainees, a number of questions will highlight some important neuropathology principles; for senior practitioners, most cases will be interesting and challenging. 